\title{
Desempenho motor. Um estudo normativo e criterial em crianças da Região Autónoma da Madeira, Portugal
}

\author{
Gil H. Afonso ${ }^{1}$ \\ Duarte L. Freitas ${ }^{2}$ \\ José M. Carmo ${ }^{3}$ \\ Johan A. Lefevre 4 \\ Maria J. Almeida ${ }^{2}$ \\ Vítor P. Lopes ${ }^{5}$ \\ Ana C. Neves ${ }^{1}$ \\ Ana J. Rodrigues ${ }^{1}$ \\ António M. Antunes ${ }^{1}$ \\ Carlos M. Esteves ${ }^{1}$ \\ Luís P. Conceição ${ }^{1}$ \\ Élvio R. Gouveia ${ }^{1}$ \\ Francisco J. Fernandes ${ }^{1}$ \\ José A. Maia ${ }^{6}$
}

https://doi.org/10.5628/rpcd.09.02-03.160

\section{RESUMO}

O objectivo central do presente estudo foi construir valores de referência para as habilidades de locomoção e de manipulação em crianças da Região Autónoma da Madeira, Portugal.

A amostra envolveu 853 crianças, 426 rapazes e 427 raparigas, com idades compreendidas entre os 3 aos 10 anos, que participaram na pesquisa 'Crescer com Saúde na Região Autónoma da Madeira'. As habilidades motoras foram avaliadas através do 'Test of Gross Motor Development'.

As crianças madeirenses apresentaram uma melhoria de resultados com a idade, na quase totalidade das habilidades motoras. Os rapazes foram mais proficientes do que as raparigas nas habilidades de manipulação. O maior número de crianças madeirenses foi classificado na categoria 'médio' nas habilidades de locomoção (51.5\%) e de manipulação (37.7\%). As crianças madeirenses apresentaram equivalentes etários abaixo da média nas habilidades de locomoção $(86.5 \%)$ e de manipulação $(87.7 \%)$. Um aumento de mestria com a idade foi observado na corrida, galope, deslocamento lateral, drible, agarrar e lançamento por cima do ombro. As crianças madeirenses apresentaram desempenhos inferiores relativamente às norte-americanas. Os resultados da presente pesquisa devem fomentar a investigação e conduzir à implementação de programas na escola e demais instituições com o objectivo de promover o normal crescimento e desenvolvimento motor das nossas crianças.

Palavras-chave: desenvolvimento motor, habilidade motora, padrão motor, $\mathrm{TGMD}_{2}$

\author{
${ }^{1}$ Secretaria Regional de Educação e Cultura \\ Região Autónoma da Madeira, Portugal \\ ${ }_{2}^{2}$ Departamento de Educação Física e Desporto, \\ Universidade da Madeira, Portugal \\ ${ }_{3}^{3}$ Departamento de Matemática e Engenharias, \\ Universidade da Madeira, Portugal \\ ${ }_{4}^{4}$ Department of Biomedical Kinesiology \\ Faculty of Kinesiology and Rehabilitation Sciences \\ Katholieke Universiteit Leuven, Bélgica \\ ${ }^{5}$ Instituto Politécnico de Bragança, Portugal \\ ${ }^{6}$ Faculdade de Desporto \\ Universidade do Porto, Portugal
}

\author{
ABSTRACT \\ Motor performance. A normative and criterial study in children \\ from the Autonomous Region of Madeira, Portugal
}

The main purpose of this study was to provide reference values for locomotor and object control skills in children from Autonomous Region of Maderia, Portugal.

The sample involved 853 individuals, 426 boys and 427 girls, from 3 to 10 year old, which participated in the 'Growth with Health in Autonomous Region of Maderia'. Motor skills were assessed by the 'Test of Gross Motor Development'.

Madeira children presented improvements with age, in almost all the fundamental motor skills. The boys were a bit more proficient than girls on the object control skills. The largest number of Madeira children was classified at the 'average' category related to the locomotor (51,5\%) and object control skills (37, 7\%). Madeira children showed age equivalents bellow the average in locomotor (86.5\%) and object control (87.7\%) skills. Increases on the mastery were observed for run, gallop, slide, stationary dribble and overhead throw. Children from Autonomous Region of Madeira presented lower results comparatively to North-American peers.

Our data should encourage the research and lead to the implementation of programmes in schools and other institutions with the purpose of promoting the normal growth and motor development of Madeira children and youth.

Key- words: motor development, skill and motor pattern, $T G M D_{2}$ 


\section{INTRODUÇÃO}

As crianças têm o potencial de aprender e desenvolver uma variedade de padrões motores e habilidades motoras mais especializadas. Tais actividades são parte integrante do seu repertório motor e fornecem um meio através do qual as crianças vivenciam muitas dimensões do seu envolvimento, especialmente em idade pré-escolar(10).

A grande variabilidade nos níveis e padrões de desenvolvimento das habilidades de locomoção e de manipulação em crianças de vários países é um facto, embora nem sempre sejam claros a extensão e o significado dos preditores da variação observada. Ulrich(21) aplicou o 'Test of Gross Motor Development' $\left(\mathrm{TGMD}_{2}\right)$ a 1208 crianças de ambos os sexos, dos 3 aos 10 anos, residentes em 4 áreas geográficas dos Estados Unidos da América. A grande maioria das crianças apresentou um desempenho motor médio e percentagens mais baixas foram encontradas nos extremos. No Brasil, Andrade et al.(2) utilizaram o $\mathrm{TGMD}_{2}$ para analisar as habilidades de locomoção e de manipulação de 33 crianças de 4 e 6 anos. Os valores médios dos equivalentes etários de locomoção e de manipulação estavam abaixo da idade cronológica. Não foram observadas diferenças com significado estatístico na idade de locomoção e idade de manipulação.

Em Portugal Continental, Saraiva et al.(16) avaliaram 151 crianças, 81 rapazes e 70 raparigas, do $1^{\circ}$ Ciclo do Ensino Básico, com idades compreendidas entre os 6 e os 9 anos, residentes em Viana do Castelo e Coimbra. A percentagem de crianças classificadas nas categorias do desempenho motor foi a seguinte: 'muito superior' (0\%), 'superior' $(0.6 \%)$, 'acima da média' (0.7\%), 'média' (25.5\%), 'abaixo da média' (16.6\%), 'fraco' (46.6\%) e 'muito fraco' (9.9\%). Em Braga, Lopes ${ }^{(7)}$ procurou avaliar as habilidades motoras de 21 crianças, 8 rapazes e 13 raparigas, com 6 e 7 anos de idade. A classificação das crianças nas habilidades de locomoção foi a seguinte: grupo 1 (22.8\%), grupo 2 (42.9\%) e grupo 3 (33.3\%). Nas habilidades de manipulação os valores foram inferiores: grupo 1 (71.4\%), grupo $2(23.8 \%)$ e grupo $3(4.8 \%)$.

Há evidência que as crianças têm vindo a apresentar um decréscimo no desempenho das suas habilidades motoras (23). Contudo, não é do nosso conhecimento a existência de qualquer informação empírica sobre os diferentes aspectos de desenvolvimento motor nas crianças madeirenses. Uma avaliação das habilidades de locomoção e de manipulação é justificada pela monitorização das mudanças que ocorrem com o crescimento físico humano e desenvolvimento motor, pela identificação das crianças com atrasos motores e pela obtenção de esclarecimentos sobre estratégias de ensino e aprendizagem. Daqui que os objectivos desta pesquisa sejam os seguintes: 1 ) construir valores de referência nas habilidades de locomoção e de manipulação; 2) analisar a variação associada à idade e ao sexo em cada habilidade motora; e 3) comparar as crianças madeirenses com outras amostras.

\section{MATERIAL E MÉTODOS \\ Amostra}

Os elementos que integram a amostra participaram no projecto de investigação 'Crescer com Saúde na Região Autónoma da Madeira' (CRES). A pesquisa apresenta um delineamento transversal incluindo 853 crianças (426 rapazes e 427 raparigas), com idades compreendidas entre os 3 e os 10 anos. A selecção dos participantes teve lugar nos 11 concelhos da RAM, ie, a totalidade dos concelhos das ilhas da Madeira e Porto Santo. A ilha da Madeira está localizada no Oceano Atlântico, a sudoeste do território de Portugal Continental entre os meridianos $16^{\circ} 39^{\prime}$ $19^{\prime \prime} \mathrm{W}$ e $17^{\circ} 15^{\prime} 54^{\prime \prime}$ W e os paralelos $32^{\circ} 37^{\prime} 52^{\prime \prime} \mathrm{N}$ e $32^{\circ} 52^{\prime} 08^{\prime \prime}$ N. A ilha do Porto Santo situa-se nos meridianos $16^{\circ} 15^{\prime} 35^{\prime \prime} \mathrm{W}$ e $16^{\circ} 24^{\prime} 35^{\prime \prime} \mathrm{W}$ e os paralelos $32^{\circ} 59^{\prime} 40^{\prime \prime} \mathrm{N}$ e $33^{\circ} 07^{\prime} 35^{\prime \prime} \mathrm{N}$. Genericamente, os elementos foram estratificados em função do local de residência, ano de escolaridade e características do edifício escolar (uma análise detalhada dos procedimentos de amostragem é descrita por Freitas et al.(5). A recolha dos dados foi efectuada por 6 licenciados em Educação Física e Desporto e teve lugar em 2006. Uma visão geral sobre a constituição da amostra é apresentada no Quadro 1. O estudo foi aprovado pela Universidade da Madeira (UMa) e pela Comissão de Ética do Hospital Central do Funchal. Todos os participantes e/ou Encarregados de Educação foram informados acerca da natureza e propósitos do estudo. Previamente, foi obtido consentimento informado para participação de todas as crianças no projecto CRES. 
Quadro 1. Número de crianças envolvidas no projecto 'Crescer com Saúde na RAM'.

\begin{tabular}{|c|c|c|c|}
\hline Idade (Anos)† & & & Total \\
\hline & Masculino & Feminino & \\
\hline 3 & 31 & 31 & 62 \\
\hline 4 & 53 & 42 & 95 \\
\hline 5 & 51 & 56 & 107 \\
\hline 6 & 54 & 59 & 113 \\
\hline 7 & 52 & 51 & 103 \\
\hline 8 & 55 & 47 & 102 \\
\hline 9 & 48 & 56 & 104 \\
\hline 10 & 82 & 85 & 167 \\
\hline Total & 426 & 427 & 853 \\
\hline
\end{tabular}

+3 anos $=3.00$ a 3.99

\section{Avaliação e classificação das crianças}

$\mathrm{O} \mathrm{TGMD}_{2}{ }^{(21)}$ foi usado na avaliação das habilidades motoras. A bateria é composta por 12 habilidades motoras divididas em dois subconjuntos: locomoção e manipulação. O primeiro avalia as habilidades motoras envolvidas no deslocamento do centro de gravidade e inclui: corrida, galope, salto a pé coxinho, salto, salto em comprimento sem corrida preparatória e deslocamento lateral. O segundo avalia as habilidades motoras associadas à projecção dos objectos e integra: batimento de uma bola com um bastão, drible, agarrar, pontapear, lançamento de uma bola por cima do ombro e lançamento de uma bola por baixo.

Genericamente, o $\mathrm{TGMD}_{2}$ fornece quatro tipos de resultados: dados brutos, percentis, valores estandardizados e equivalentes etários. Os dados brutos constituem o total de pontos obtidos em cada teste/habilidade motora. Os percentis indicam a posição de um indivíduo na distribuição. Por exemplo, se uma criança se encontra no P5 significa que 95\% dos indivíduos do seu grupo e sexo apresentam valores mais elevados do que ele ou, por outro lado, apenas $5 \%$ da amostra possui um valor igual ou inferior ao dele. Os valores estandardizados fornecem a indicação mais clara de performance num dado subconjunto (locomoção e/ou manipulação). O quocien- te motor $(\mathrm{QM})$ é um outro tipo de valor estandardizado, um compósito dos resultados dos dois subconjuntos, representando a performance total da criança. Os equivalentes etários relacionam o resultado obtido com a idade, i.e., uma 'medida do nível de desenvolvimento' da criança.

A recolha e posterior quantificação dos dados obedecem a um trajecto específico. Primeiro, a criança dispõe de duas tentativas para realizar cada habilidade motora. Na avaliação das habilidades motoras há uma decomposição ou segmentação das mesmas em três, quatro ou cinco componentes/critérios de êxito. Se a criança executa correctamente a componente/critério é atribuído o valor 1; se não, é atribuído o valor 0 . A soma dos resultados das duas tentativas, nas componentes e/ou critérios de êxito conduz a um valor bruto em cada habilidade motora. A soma dos resultados das 6 habilidades motoras, em cada subconjunto, dá origem a uma pontuação para as habilidades de locomoção e a uma pontuação para as habilidades de manipulação. Segundo, os dois subconjuntos de valores brutos são convertidos em valores estandardizados, a partir de uma distribuição com média 10 e um desvio padrão de 3, usando tabelas construídas para o efeito. Os valores estandardizados são combinados e convertidos num quociente motor (QM) que permite classificar as crianças em 7 categorias: muito superior, superior, acima da média, médio, abaixo da média, fraco e muito fraco. Terceiro, a mestria das crianças, em cada habilidade motora ou componente/critério de êxito, é calculada a partir do sucesso nas duas tentativas.

\section{Preparação da equipa de campo e estudo piloto}

Um dos coordenadores do CRES efectuou contactos com o Prof. Doutor Dale Ulrich, autor do $\mathrm{TGMD}_{2}$, a propósito de aspectos particulares da bateria e procedimentos de avaliação/materiais. Na fase seguinte, a bateria de testes foi apresentada aos elementos da equipa de campo. Conhecidos os testes, realizaram-se várias sessões de observação e treino no Laboratório de Crescimento e Desenvolvimento da UMa.

Os elementos da equipa de campo e de investigação realizaram um estudo piloto em 46 alunos do Ensino Pré-Escolar (3-5 anos) e do $1^{\circ}$ Ciclo do Ensino Básico (6-9 anos) da Escola Básica do $1^{\circ}$ Ciclo, com Ensino 
Quadro 2. Coeficiente de correlação intra-classe [R], intervalo de confiança 95\% (IC 95\%) e erro padrão de medição (EPM): estudo piloto.

\begin{tabular}{|c|c|c|c|c|}
\hline $\mathrm{TGMD}_{2}$ & $\mathrm{n}$ & $\mathrm{R}$ & IC 95\% & EPM \\
\hline \multicolumn{5}{|l|}{ Locomoção } \\
\hline Corrida & 46 & 0.769 & $0.583-0.872$ & 0.806 \\
\hline Galope & 45 & 0.945 & $0.901-0.970$ & 0.738 \\
\hline Salto a pé coxinho & 45 & 0.851 & $0.720-0.918$ & 0.917 \\
\hline Salto (transposição de um objecto) & 46 & 0.770 & $0.584-0.873$ & 0.812 \\
\hline Salto em comp. s/ corrida preparatória & 46 & 0.649 & $0.365-0.806$ & 0.925 \\
\hline Deslocamento lateral $\dagger$ & 46 & - & - & - \\
\hline \multicolumn{5}{|l|}{ Manipulação } \\
\hline Batimento de uma bola com um bastão & 46 & 0.700 & $0.457-0.834$ & 1.099 \\
\hline Drible & 46 & 0.945 & $0.900-0.969$ & 0.651 \\
\hline Agarrar & 46 & 0.731 & $0.513-0.851$ & 0.603 \\
\hline Pontapear & 46 & 0.748 & $0.545-0.861$ & 0.837 \\
\hline Lançamento por cima & 46 & 0.780 & $0.602-0.878$ & 1.002 \\
\hline Lançamento por baixo & 46 & 0.693 & $0.444-0.830$ & 0.924 \\
\hline
\end{tabular}

†Face à reduzida variação no desempenho do deslocamento lateral entre o teste e o reteste, optamos pelo recurso ao teste t de medidas repetidas cujos resultados são os seguintes: $t_{(45)}=-1,157 ; p=0.249$. Salto em comp. $s$ / corrida preparatória = salto em comprimento sem corrida preparatória.

Pré-Escolar, dos Ilhéus. O procedimento teste-reteste foi utilizado para estimar a fiabilidade dos resultados de avaliação. A primeira avaliação decorreu no dia 6 de Dezembro de 2005 e a segunda avaliação no dia 15 de Dezembro do mesmo ano. Cada membro da equipa de campo administrou apenas dois testes, um de locomoção e outro de manipulação. Os coeficientes de correlação intra-classe (R) para o estudo piloto são apresentados no Quadro 2.

Os valores de $\mathrm{R}$ são superiores a 0.70 , na maioria das habilidades motoras, o que mostra a elevada precisão dos registos de avaliação. Excepções foram observadas no salto em comprimento sem corrida preparatória (0.65) e no lançamento da bola por baixo (0.69). Contudo, os valores estão muito próximos dos mínimos aceitáveis sugeridos por Ulrich(21) e Safrit(15). Estes resultados conduziram a uma dupla orientação: (1) maior rigor na avaliação do salto em comprimento sem corrida preparatória e do lançamento da bola por baixo e (2) alargar o período de treino na avaliação destas habilidades motoras.

\section{Procedimentos estatísticos}

A média e o desvio padrão foram utilizados na descrição das habilidades motoras. A fiabilidade testereteste foi estimada na base do coeficiente de correlação intraclasse. O teste $t$ para duas amostras independentes foi usado para aferir as diferenças entre sexos em cada habilidade motora.

O cálculo do valor bruto para cada habilidade motora; a soma dos valores brutos no subconjunto de locomoção e de manipulação; a conversão dos valores brutos em percentis e valores estandardizados; a combinação e conversão da soma dos valores estandardizados em percentis e quocientes; a classificação das crianças nas 7 categorias; a conversão dos valores brutos em equivalentes etários, bem como a percentagem de mestria em cada habilidade motora e/ou componente/critério foram efectuados num programa construído no Mathematica, versão 5.0(23), de acordo com as indicações existentes no manual do TGMD2. 
Figura 1. Representação gráfica das habilidades de locomoção: corrida, galope, salto a pé coxinho (salto $P C$ ), salto, salto em comprimento sem corrida preparatória [SCP] e deslocamento lateral (DesL).
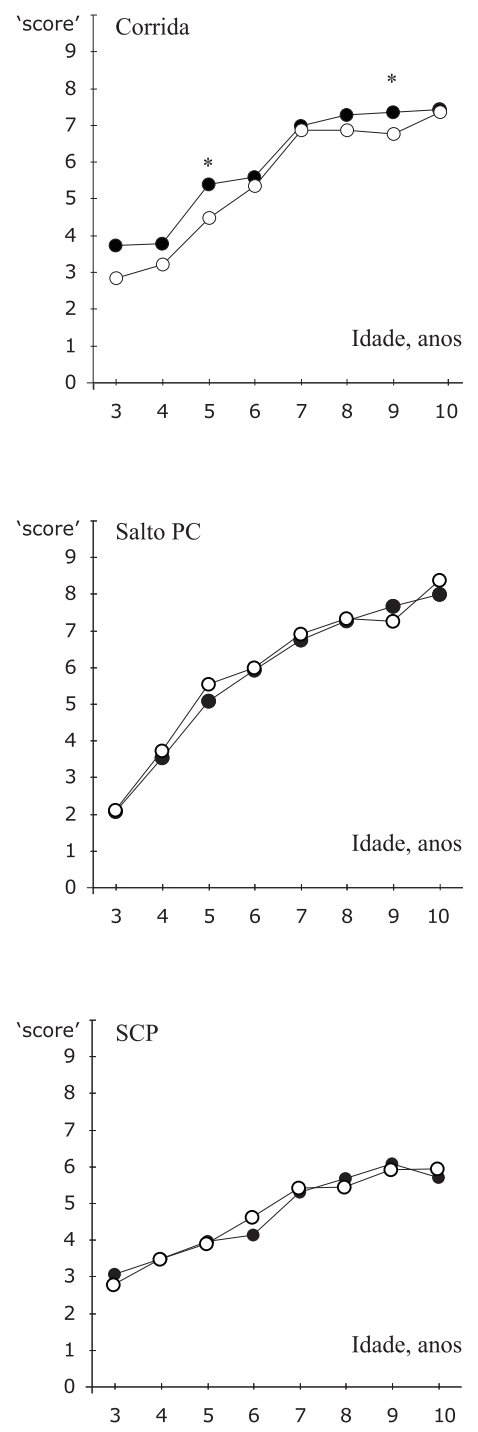

$\longrightarrow$ Rapazes
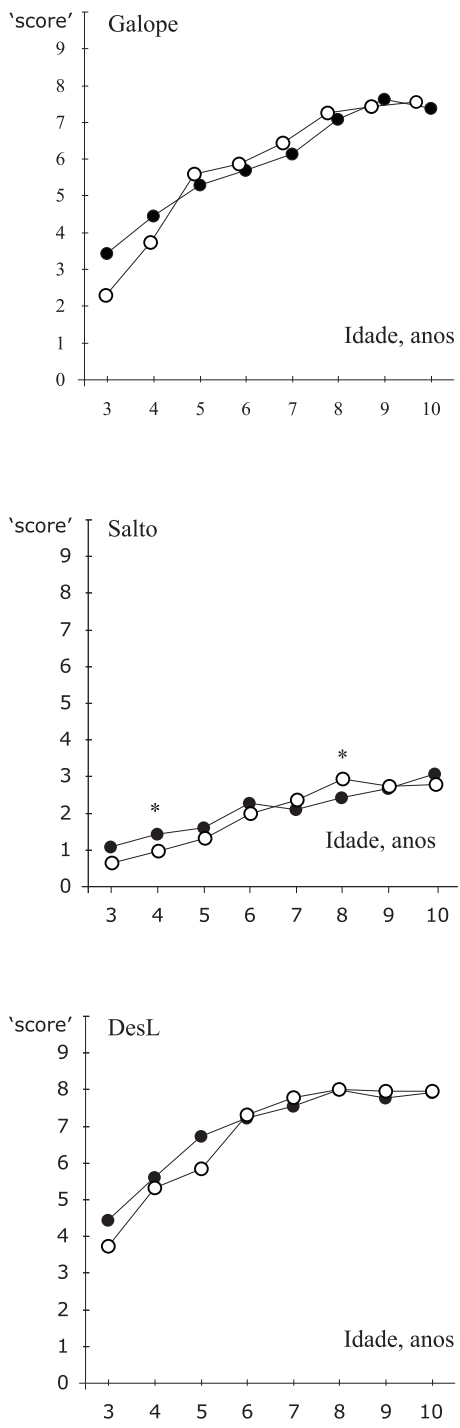

-
As restantes análises foram efectuadas a partir do recurso ao 'Statistical Analysis System Program'(17). O significado estatístico foi mantido em $\mathrm{p}<0.05$.

\section{RESULTADOS}

Valores descritivos para cada habilidade motora e dimorfismo sexual

Os valores médios nas habilidades de locomoção e de manipulação, por idade e sexo, são representados graficamente nas Figuras 1 e 2. É evidente uma melhoria dos resultados com a idade na quase totalidade das habilidades motoras e nos dois sexos. Ao nível das habilidades de locomoção, ganhos mais elevados são observados no galope e no salto a pé coxinho. 

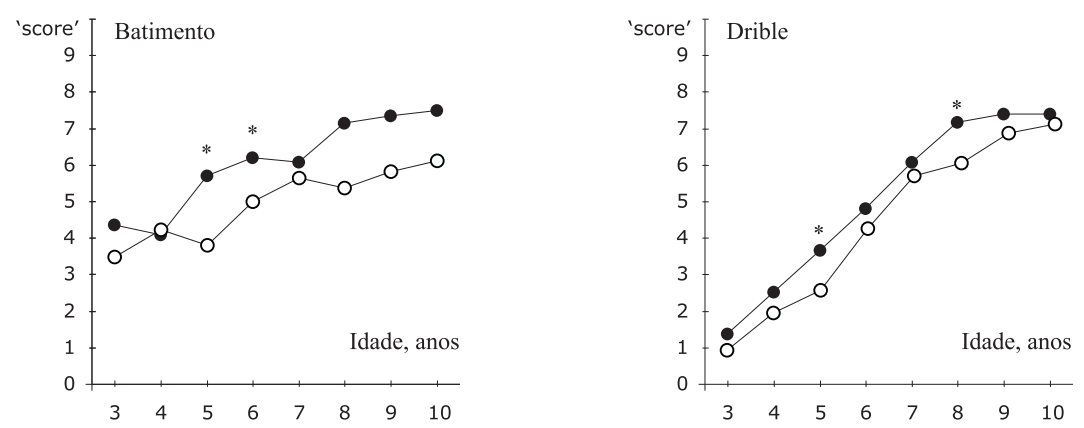

Figura 2. Representação gráfica das habilidades de manipulação: batimento de uma bola estática, drible, agarrar, pontapear, lançamento de uma bola por cima do ombro (lançamento $S B$ ) e lançamento de uma bola por baixo (lançamento $P B$ ).
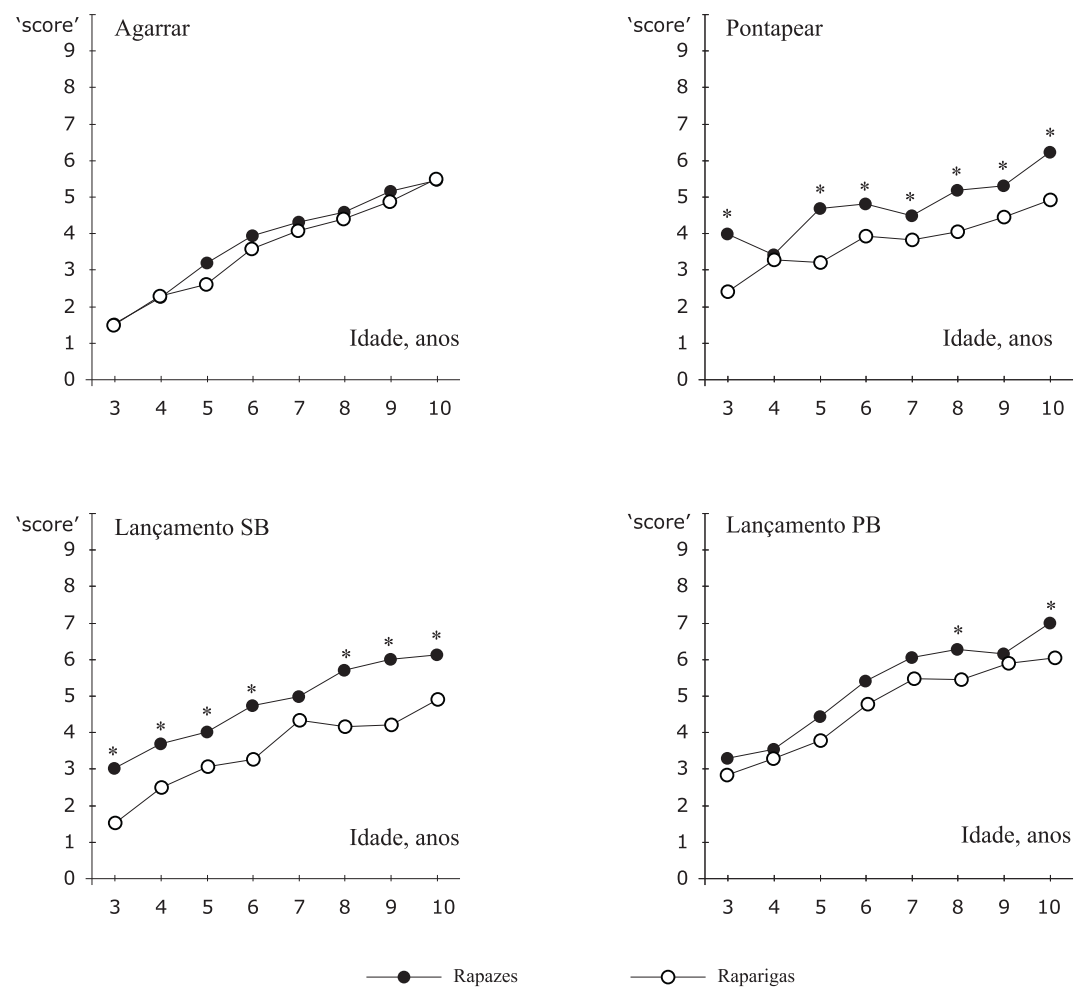

As diferenças com significado estatístico entre rapazes e raparigas estão assinaladas com um asterisco sobre o intervalo etário correspondente. Os valores médios dos rapazes são mais elevados do que os das raparigas e alcançam significado estatístico na corrida (5 e 9 anos) e no salto (4 anos). As raparigas apresentam melhores desempenhos no salto, aos 8 anos. No subconjunto das habilidades de manipulação, ganhos mais pronunciados, ao longo da idade, são observados no drible e no lançamento por baixo.
Os rapazes apresentam melhores desempenhos do que as raparigas, na quase totalidade das habilidades motoras e intervalos etários. Diferenças com significado estatístico, favorecendo os rapazes, são claramente observadas no pontapear e no lançamento da bola por cima do ombro. Os rapazes são ainda mais proficientes no batimento de uma bola estática (5 e 6 anos), drible (5 e 8 anos) e lançamento da bola por baixo (8 e 10 anos). 
Quadro 3. Equivalentes etários: locomoção e manipulação.

\begin{tabular}{|c|c|c|c|c|c|c|}
\hline \multirow{3}{*}{ Equivalentes etários } & \multicolumn{4}{|c|}{ Sexo } & & \\
\hline & \multicolumn{2}{|c|}{ Masculino } & \multicolumn{2}{|c|}{ Feminino } & \multicolumn{2}{|c|}{ Total } \\
\hline & $\mathrm{n}$ & $\%$ & $\mathrm{n}$ & $\%$ & $\mathrm{n}$ & $\%$ \\
\hline \multicolumn{7}{|l|}{ Locomoção } \\
\hline Acima da idade cronológica & 64 & 15.0 & 51 & 12.0 & 115 & 13.5 \\
\hline Igual à idade cronológica & - & - & - & - & - & - \\
\hline Abaixo da idade cronológica & 362 & 84.9 & 373 & 88.0 & 735 & 86.5 \\
\hline \multicolumn{7}{|l|}{ Manipulação } \\
\hline Acima da idade cronológica & 48 & 11.3 & 56 & 13.2 & 104 & 12.2 \\
\hline Igual à idade cronológica & 1 & 0.2 & - & - & 1 & 0.1 \\
\hline Abaixo da idade cronológica & 377 & 88.5 & 368 & 86.8 & 745 & 87.7 \\
\hline
\end{tabular}

Quadro 4. Classificação das crianças: locomoção, manipulação e quociente motor.

\begin{tabular}{|c|c|c|c|c|c|c|}
\hline \multirow[t]{2}{*}{ Classificação } & \multicolumn{2}{|c|}{ Locomoção } & \multicolumn{2}{|c|}{ Manipulação } & \multicolumn{2}{|c|}{ Quociente motor } \\
\hline & $\mathrm{n}$ & $\%$ & $\mathrm{n}$ & $\%$ & $\mathrm{n}$ & $\%$ \\
\hline Excelente & - & - & - & - & 1 & 0.1 \\
\hline Superior & 1 & 0.1 & 4 & 0.5 & 4 & 0.5 \\
\hline Acima da média & 17 & 2.0 & 11 & 1.3 & 9 & 1.1 \\
\hline Média & 438 & 51.5 & 320 & 37.7 & 241 & 28.4 \\
\hline Abaixo da média & 263 & 30.9 & 285 & 33.5 & 265 & 31.2 \\
\hline Fraco & 109 & 12.8 & 181 & 21.3 & 260 & 30.6 \\
\hline Muito fraco & 22 & 2.6 & 49 & 5.8 & 70 & 8.2 \\
\hline
\end{tabular}

\section{Equivalentes etários nas habilidades} de locomoção e de manipulação

Os equivalentes etários nas habilidades de locomoção e de manipulação são apresentados no Quadro 3. Nas habilidades de locomoção há uma grande percentagem de rapazes $(84.9 \%)$ e raparigas $(88.0 \%)$ que se encontra abaixo da idade cronológica. O valor total é de $86.5 \%$. As percentagens de crianças acima da idade cronológica são $15.0 \%$ (rapazes), $12.0 \%$ (raparigas) e $13.5 \%$ (total). Nas habilidades de manipulação, os resultados são similares aos anteriores: $88.5 \%$ dos rapazes, $86.8 \%$ das raparigas e $87.7 \%$ no total encontram-se abaixo da idade cronológica. As percentagens correspondentes para a categoria acima da idade cronológica são: $11.3 \%$ (rapazes), $13.2 \%$ (raparigas) e $12.2 \%$ (total). A percentagem de crianças cuja idade cronológica é igual ao equivalente etário é muito reduzida $[0.2 \%$ (rapazes) $]$ ou nula nas habilidades de locomoção e de manipulação.

\section{Classificação das crianças nas habilidades de locomoção e de manipulação}

A classificação dos elementos da amostra da RAM é apresentada no Quadro 4. O maior aglomerado de crianças encontra-se na categoria média: locomoção (51.5\%), manipulação (37.7\%) e quociente motor (28.4\%). Logo de seguida, as crianças madeirenses são maioritariamente classificadas nas categorias 
Quadro 5. Percentagem de crianças demonstrando mestria na bateria $T G M D_{2}$.

\begin{tabular}{|c|c|c|c|c|c|c|c|c|}
\hline \multirow[t]{2}{*}{ TGMD2 } & \multicolumn{8}{|c|}{ Idade } \\
\hline & 3 & 4 & 5 & 6 & 7 & 8 & 9 & 10 \\
\hline \multicolumn{9}{|l|}{ Locomoção } \\
\hline Corrida & 3 & 3 & 11 & 16 & 55 & 60 & 62 & 73 \\
\hline Galope & 13 & 21 & 43 & 45 & 52 & 73 & 86 & 78 \\
\hline Salto a pé coxinho & - & 2 & 2 & 4 & 10 & 14 & 17 & 29 \\
\hline Salto (transposição de um objecto] & - & - & - & 2 & 1 & 3 & 2 & $?$ \\
\hline Salto em comp. s/ corrida preparatória & - & 2 & 3 & $?$ & 21 & 19 & 27 & 19 \\
\hline Deslocamento lateral & 25 & 52 & 59 & 83 & 87 & 98 & 94 & 96 \\
\hline \multicolumn{9}{|l|}{ Manipulação } \\
\hline Batimento bola estática com um bastão & 2 & - & - & 3 & 5 & 6 & 16 & 19 \\
\hline Drible & 2 & 4 & 9 & 12 & 38 & 55 & 64 & 67 \\
\hline Agarrar & 2 & 1 & 2 & 16 & 20 & 24 & 43 & 67 \\
\hline Pontapear & 2 & 2 & 4 & 1 & 2 & 2 & 4 & 24 \\
\hline Lançamento por cima & - & 2 & 2 & 4 & 7 & 11 & 7 & 15 \\
\hline Lançamento por baixo & 2 & 2 & 6 & 7 & 20 & 18 & 31 & 38 \\
\hline
\end{tabular}

Salto em comp. $\mathrm{s} /$ corrida preparatória = salto em comprimento sem corrida preparatória; batimento bola estática com um bastão = batimento de uma bola estática com um bastão.

'abaixo da média e fraco'. A percentagem de crianças no limite inferior da escala 'muito fraco' e à direita da distribuição 'acima da média, superior, superior e muito superior’ é baixa.

\section{Percentagem de crianças demonstrando mestria no $\mathrm{TGMD}_{2}$}

A percentagem de crianças que alcançaram a mestria nas habilidades de locomoção e de manipulação é apresentada no Quadro 5. Na corrida, galope e deslocamento lateral é observada uma maior mestria com o avanço da idade, à excepção do intervalo etário 8-10 anos. No salto a pé coxinho, salto e salto em comprimento sem corrida preparatória, a percentagem de mestria é reduzida, mesmo nos intervalos etários finais. No subconjunto das habilidades de manipulação, melhorias expressivas com a idade são observadas no drible, agarrar e lançamento por baixo. No batimento de uma bola estática, no pontapear e no lançamento por cima não são observadas grandes alterações com a idade.
Quanto à mestria, o Quadro 6 apresenta as percentagens alcançadas nas componentes/critérios de êxito para cada habilidade motora. Ao nível da corrida há um aumento da mestria ao longo da idade nas quatro componentes. Um padrão de corrida menos desenvolvido é observado na componente/critério dois, até aos 5 anos (máximo de $28 \%$ ) e na componente três, até aos 4 anos (máximo de 25\%). A mestria no galope aumenta linearmente com a idade na totalidade das componentes/critérios, observando-se esporadicamente algumas inversões nos valores alcançados.

No salto a pé coxinho, o valor percentual da mestria aumenta, ao longo da idade, nas cinco componentes/critérios. Contudo, os ganhos percentuais na primeira componente são reduzidos e, aos 10 anos, apenas $53 \%$ dos madeirenses apresentam mestria. No salto, a melhoria de mestria ao longo da idade é observada apenas na primeira componente. As percentagens de mestria nas restantes componentes/critérios são baixas. 


\section{Teste/critérios}

\section{Corrida}

\begin{tabular}{|c|c|c|c|c|c|c|c|c|}
\hline Os braços movem-se em oposição às pernas, com os cotovelos flectidos & 53 & 50 & 66 & 71 & 83 & 88 & 84 & 86 \\
\hline Breve período aéreo (os dois pés não estão em contacto com o solo] & 12 & 17 & 28 & 44 & 85 & 90 & 87 & 91 \\
\hline Redução da superfície de apoio ( $1^{\circ}$ o calcanhar e depois os dedos $)$ & 25 & 25 & 54 & 60 & 79 & 78 & 86 & 95 \\
\hline Perna livre realiza um ângulo de aproximadamente $90^{\circ}$ & 67 & 67 & 88 & 88 & 92 & 93 & 89 & 92 \\
\hline
\end{tabular}

\section{Galope}

Braços flectidos elevados ao nível da cintura no início do movimento

\begin{tabular}{llllllllll}
\hline Passo à frente, traz o outro pé para uma posição adjacente ou lig. avançada & 38 & 57 & 75 & 71 & 85 & 96 & 98 & 97 \\
\hline
\end{tabular}

\begin{tabular}{|c|c|c|c|c|c|c|c|c|}
\hline Período breve em que os pés não estão em contacto com o solo & 48 & 63 & 78 & 82 & 85 & 96 & 97 & 93 \\
\hline Mantém um padrão rítmico para os quatro galopes suc & 27 & 40 & 65 & 60 & 60 & 81 & 89 & 87 \\
\hline
\end{tabular}

\section{Salto a pé coxinho}

\begin{tabular}{|c|c|c|c|c|c|c|c|c|}
\hline A perna livre é utilizada como um pêndulo para produzir força & 8 & 18 & 22 & 19 & 29 & 29 & 39 & 53 \\
\hline A perna livre está flectida e atrás do corpo & 28 & 38 & 57 & 55 & 61 & 69 & 58 & 72 \\
\hline Os braços estão flectidos e deslocam-se para a frente para produzir força & 10 & 15 & 15 & 20 & 39 & 43 & 56 & 69 \\
\hline É capaz de realizar três saltos a pé coxinho com o pé não predilecto & 13 & 24 & 57 & 77 & 88 & 97 & 97 & 95 \\
\hline É capaz de realizar três saltos a pé coxinho com o pé predilecto & 40 & 65 & 92 & 97 & 96 & 100 & 98 & 97 \\
\hline
\end{tabular}

\section{Salto}

Impulsão com um pé e recepção com o outro

\begin{tabular}{|c|c|c|c|c|c|c|c|c|}
\hline Impulsão com um pé e recepção com o outro & 25 & 39 & 46 & PO & 75 & 84 & 85 & 81 \\
\hline Fase aérea. Pés estão afastados a uma maior distância do que na corrida & - & 2 & - & 10 & 5 & 9 & $?$ & 28 \\
\hline Braço oposto à perna de ataque aproxima-se da perna & - & 3 & 1 & 4 & 10 & 15 & 15 & 19 \\
\hline
\end{tabular}

\section{Salto em comprimento sem corrida preparatória}

\begin{tabular}{|c|c|c|c|c|c|c|c|c|}
\hline Flexão dos joelhos e colocação dos braços atrás & 13 & 21 & 28 & 37 & 55 & 64 & 77 & 72 \\
\hline Projecção dos braços para a frente e para cima (fase final acima da cabeça) & - & 3 & 9 & 12 & 26 & 26 & 32 & 29 \\
\hline Impulsão e recepção a dois pés e em simultâneo & 87 & 91 & 93 & 94 & 93 & 94 & 95 & 96 \\
\hline Braços dirigidos para o solo & 37 & 40 & 51 & 57 & 75 & 77 & 79 & 77 \\
\hline
\end{tabular}

\section{Deslocamento lateral}

\begin{tabular}{|c|c|c|c|c|c|c|c|c|}
\hline Corpo na lateral e ombros alinhados com o solo (linha) & 60 & 74 & 81 & 97 & 99 & 100 & 96 & 100 \\
\hline Passo lateral e deslocamento do outro pé para próximo do apoio & 52 & 74 & 88 & 91 & 94 & 98 & 99 & 100 \\
\hline Mínimo de quatro ciclos contínuos para a direita & 50 & 64 & 74 & 87 & 95 & 100 & 98 & 98 \\
\hline Mínimo de quatro ciclos contínuos para a esquerda & 47 & 58 & 64 & 84 & 92 & 100 & 98 & 96 \\
\hline
\end{tabular}


Quadro 7. Percentagem de crianças demonstrando mestria nas habilidades de manipulação $\left(\operatorname{TGMD}_{2}\right)$ : componentes/critério.

\section{Teste/critérios}

Idade

$\begin{array}{llllllll}3 & 4 & 5 & 6 & 7 & 8 & 9 & 10\end{array}$

\section{Batimento numa bola estática com um bastão}

\begin{tabular}{|c|c|c|c|c|c|c|c|c|}
\hline Mão dominante segura o bastão em cima da mão não dominante & 63 & 71 & 67 & 81 & 84 & 87 & 89 & 92 \\
\hline Lado não dominante do corpo está mais próximo da bola; pés paralelos & 37 & 40 & 55 & 71 & 82 & 92 & 88 & 93 \\
\hline Rotação do ombro e da anca durante o batimento & 13 & 14 & 15 & 22 & 31 & 35 & 46 & 52 \\
\hline Transfere do peso do corpo para a perna da frente & 8 & 4 & 9 & 9 & 11 & 12 & 23 & 23 \\
\hline 0 bastão toca na bola & 50 & 45 & 60 & 64 & 45 & 55 & 54 & 52 \\
\hline
\end{tabular}

\section{Drible (no lugar)}

\begin{tabular}{|c|c|c|c|c|c|c|c|c|}
\hline Braços flectidos elevados ao nível da cintura no início do movimento & 7 & 21 & 17 & 24 & 41 & 66 & 72 & 78 \\
\hline Passo à frente, traz o outro pé para uma posição adjacente ou lig. avançada & 3 & 16 & 23 & 41 & 63 & 70 & 85 & 83 \\
\hline Período breve em que os pés não estão em contacto com o solo & 30 & 54 & 70 & 87 & 97 & 98 & 98 & 99 \\
\hline Mantém um padrão rítmico para os quatro galopes sucessivos & 8 & 14 & 31 & 50 & 75 & 87 & 93 & 96 \\
\hline
\end{tabular}

\section{Agarrar}

Mãos à frente do corpo e flexão dos braços pelos cotovelos

\begin{tabular}{|c|c|c|c|c|c|c|c|c|}
\hline Extensão dos braços em direcção à bola & 20 & 47 & 66 & 81 & 96 & 97 & 100 & 100 \\
\hline Agarrar a bola apenas com as mãos & 2 & 6 & 8 & 23 & 35 & 45 & 64 & 78 \\
\hline
\end{tabular}

\section{Pontapear}

\begin{tabular}{|c|c|c|c|c|c|c|c|c|}
\hline Aproximação rápida e contínua à bola & 27 & 38 & 58 & 68 & 57 & 63 & 75 & 75 \\
\hline Passo alongado ou pequeno salto antes do contacto com a bola & 12 & $?$ & 9 & 13 & 18 & 14 & 17 & 38 \\
\hline Pé de apoio ao lado ou ligeiramente atrás da bola & 15 & 14 & 9 & 12 & $?$ & 15 & 20 & 41 \\
\hline Pontapear a bola com o pé preferido (peito ou ponta do pé) & 82 & 76 & 79 & 87 & 85 & 96 & 93 & 93 \\
\hline
\end{tabular}

\section{Lançamento por cima do ombro}

\begin{tabular}{|c|c|c|c|c|c|c|c|c|}
\hline Iniciar o lançamento com o movimento descendente do braço oposto & - & 6 & 7 & 15 & 19 & 16 & 14 & 20 \\
\hline Rotação da anca e do ombro atrás (ombro oposto dirigido para a parede) & 38 & 36 & 49 & 51 & 65 & 67 & 68 & 83 \\
\hline Transfere do peso corporal para o pé de apoio & 38 & 57 & 58 & 49 & 68 & 78 & 75 & 82 \\
\hline Lançamento diagonal com rotação do ombro e da anca & 22 & 38 & 41 & 63 & 68 & 74 & 74 & 81 \\
\hline
\end{tabular}

\section{Lançamento por baixo}

\begin{tabular}{|c|c|c|c|c|c|c|c|c|}
\hline Colocação da mão em baixo e atrás das costas; ombros alinhados & 37 & 41 & 52 & 64 & 83 & 86 & 84 & 88 \\
\hline Passos à frente com o pé contrário à mão de lançamento & 3 & 3 & 12 & 19 & 37 & 29 & 46 & 55 \\
\hline Pernas flectidas pelo joelho & 60 & 65 & 79 & 89 & 92 & 99 & 92 & 99 \\
\hline Largar a bola junto ao solo para que não saltite mais de $10,16 \mathrm{~cm}$ do solo & 42 & 42 & 36 & 52 & 48 & 48 & 58 & 65 \\
\hline
\end{tabular}


Paralelamente, para o salto em comprimento sem corrida preparatória, a mestria tende a aumentar com a idade na primeira, segunda e quarta componente. No deslocamento lateral, o comportamento dos resultados ao longo da idade segue um trajecto similar à maioria das habilidades motoras anteriores. A percentagem de mestria alcançada pelas crianças madeirenses nas habilidades de manipulação é apresentada no Quadro 7. Para o batimento numa bola estática com um bastão, a melhoria na mestria com a idade é claramente observada na $2^{\mathrm{a}}$ e $3^{\mathrm{a}}$ componentes. Valores percentuais elevados são observados na primeira componente. Ao nível da quarta componente, a percentagem de mestria observada na nossa amostra é muito reduzida: $8 \%$ aos 3 anos e $23 \%$ aos 10 anos. Valores percentuais muito idênticos são observados na quinta componente na totalidade do intervalo etário. Para o drible, há um aumento dos valores percentuais ao longo da idade nas quatro componentes.

Melhorias expressivas com a idade são também observadas na mestria do agarrar. Ao nível da segunda componente cerca de $96 \%$ das crianças madeirenses alcançam êxito, nas duas tentativas, aos 7 anos. Valores percentuais muito baixos são observados na terceira componente aos 3-5 anos (2\%-8\%). Para o pontapear, um aumento linear na mestria é observado na primeira componente; enquanto na quarta, os valores percentuais são já elevados aos 3 anos. No lançamento da bola por cima do ombro, melhorias na mestria com a idade são observadas na segunda, terceira e quarta componente. As crianças madeirenses alcançam percentagens de mestria muito baixas na primeira componente. Finalmente, para o lançamento da bola por baixo, os ganhos na mestria com a idade são observados na totalidade das componentes/critérios. Um aumento mais pronunciado é observado na primeira e terceira componente. Um padrão ligeiramente irregular é observado na quarta componente/critério.

\section{DISCUSSÃO}

Interpretar os resultados é uma tarefa complexa devido ao número reduzido de pesquisas que utilizaram o $\mathrm{TGMD}_{2}$, à inexistência de protocolos estandardizados de recolha da informação, e à dupla aproximação às habilidades motoras ou seja, o processo e o produto. Genericamente, o processo e o produto de uma performance motora estão positivamente relacionados. Isto é, bons resultados em termos de produto demonstram proficiência no processo de movimento, e vice-versa. É nesta base que assenta a nossa discussão.

Os resultados da presente pesquisa são parcialmente suportados por alguns 'clássicos' da literatura. Sinclair(19) estudou o desenvolvimento de várias habilidades motoras em crianças norte-americanas, dos 2 aos 6 anos de idade. O desempenho aumentou, em média, com o avanço da idade, na maioria das habilidades motoras (agarrar, correr, deslocamento lateral, galope, lançamento, pontapear e salto em comprimento sem corrida preparatória). Similarmente, Morris et al.(11) examinaram a relação entre a idade e o desempenho motor em crianças norte-americanas dos 3 aos 6 anos. No total, uma melhoria de resultados com a idade foi observada no agarrar, corrida, salto em comprimento sem corrida preparatória e lançamento de uma bola de ténis. Nelson et al.(13) observaram uma melhoria na performance de lançamento em 26 crianças (5-8 anos) seguidas ao longo de um período de 3 anos. Em Portugal, Saraiva et al.(16) observaram desempenhos irregulares: aumento nas habilidades de locomoção dos 6 aos 7 anos, seguido de um decréscimo aos 8 e aos 9 anos, enquanto, nas habilidades de manipulação, os desempenhos decresceram com a idade. As diferenças entre sexos nas habilidades de locomoção e de manipulação observadas nas crianças madeirenses são paralelas a outros trabalhos. Gabbard(6) refere que 'aos 3 anos as crianças do sexo masculino tendem a ser mais proficientes do que as raparigas em tarefas como a corrida, lançamento e salto em comprimento sem corrida preparatória. As crianças do sexo feminino, por outro lado, tendem a ser mais proficientes no salto a pé coxinho e tarefas que requerem controlo motor fino, equilíbrio e flexibilidade'. Ainda neste âmbito, Morris et al.(11) investigaram a associação da idade e sexo à performance. Os rapazes foram mais proficientes do que as raparigas no teste de lançamento. Diferenças de menor grandeza entre sexos foram também encontradas na corrida e no salto em comprimento sem corrida preparatória, onde a performance dos rapazes foi superior à das raparigas. Similarmente, Nelson et al.(12) 
observaram que os rapazes lançavam mais longe do que as raparigas e exibiam formas de lançamento mais 'maduras' aos 5 anos de idade. A performance de lançamento das raparigas foi apenas de $57 \%$ relativamente à performance dos rapazes, mas quando a performance foi ajustada por um compósito de variáveis biológicas, a performance das raparigas aumentou para $60 \%$ comparativamente à performance apresentada pelos rapazes.

As características de crescimento físico humano, o envolvimento e a sua interacção são as fontes potenciais para explicar o dimorfismo sexual observado nas habilidades de manipulação, na amostra madeirense. A este respeito, Thomas e French(20), num estudo de meta-análise observaram que os rapazes (3-7 anos) excederam as raparigas nos desempenhos relativos á velocidade de lançamento em cerca de 1.5 unidades de desvio padrão e que a diferença aumentava para 3.5 unidades de desvio padrão aos 12 anos. Tal facto conduziu os autores a sugerirem que as diferenças entre sexos no lançamento por cima do ombro têm origem em diferenças biológicas. Esta sugestão é reforçada por Malina et al.(10) e Malina(8, 9), ao referirem que as diferenças biológicas entre sexos na infância são reduzidas, mas os rapazes têm mais massa isenta de gordura e menos gordura corporal do que as raparigas. Malina ${ }^{(9)}$ refere ainda que os rapazes apresentam um rácio 'diâmetro biacromial sobre o diâmetro bicristal' maior no final da infância.

Concomitantemente, as características de envolvimento são determinantes nas diferenças entre sexos observadas na performance motora. Regra geral, os pais "olham" os filhos de forma diferente das filhas e esperam comportamentos distintos ${ }^{(10)}$.

Paralelamente, os rapazes em idade pré-escolar são mais activos do que as raparigas ${ }^{(4)}$.

A grande variabilidade observada no desempenho das habilidades de locomoção e de manipulação das crianças madeirenses é comum na literatura. Malina et al.(10) referem que a variação no desenvolvimento motor é considerável durante a infância no mesmo indivíduo, entre indivíduos e ao longo da idade. As crianças em idade pré-escolar mostram frequentemente uma tendência para um bom desempenho num dado momento e um mau desempenho no momento seguinte. A variação na performance entre períodos de avaliação distintos reflecte a variação na maturação neuromuscular, mudanças no tamanho e proporções, oportunidade para a prática e motivação. Para além disso, a avaliação de crianças exige cooperação, a qual é um factor que afecta a performance. A alternância de padrões de movimento 'maduros' numa dada idade e padrões 'imaturos' numa idade subsequente ocorrem frequentemente sem uma causa conhecida e podem reflectir variabilidade normal. De igual modo, podem estar relacionados com o desenvolvimento e aprendizagem de novas habilidades motoras, pelo que quando um padrão 'maduro' é alcançado numa tarefa, a criança pode tentar outros movimentos que minimizem o padrão 'maduro' original $(3,10)$.

Os equivalentes etários indicam o nível de desenvolvimento ou idade que corresponde um valor bruto. Uma criança com um equivalente etário acima da média, significa que o seu valor bruto é característico de uma idade cronológica mais avançada. Por outras palavras, está avançado para a sua idade cronológica. O oposto é verdadeiro para uma criança que está localizada abaixo da média. As crianças madeirenses apresentaram-se maioritariamente abaixo da média nas habilidades de locomoção e de manipulação. A única pesquisa que encontramos na literatura com equivalentes etários foi desenvolvida na Universidade Estadual de Londrina. Andrade et al.(2) referem valores médios nos equivalentes etários de locomoção e de manipulação abaixo da idade cronológica. Estes resultados são paralelos à amostra madeirense. Devido à interpolação, extrapolação e ajustamento usados na criação dos equivalentes etários no método original, Ulrich ${ }^{(21)}$ sugere alguma prudência na interpretação dos resultados.

Uma grande fatia de crianças madeirenses é classificada nas categorias 'abaixo da média', 'fraco' e 'muito fraco'. Esta partição difere da amostra norteamericana $^{(21)}$. As crianças norte-americanas estão mais circunscritas às categorias 'médio' e acima da média. Em oposição, desempenhos mais fracos do que os que foram observados nos rapazes e raparigas madeirenses foram encontrados no Brasil(1). Em Portugal Continental, Saraiva et al.(16) observaram valores percentuais muito próximos à amostra madeirense, à excepção da categoria 'fraco', na qual os madeirenses quase duplicam a percentagem dos 
seus pares. Resultados similares à amostra madeirense foram também observados em crianças bracarenses ${ }^{(7)}$.

Ao nível da mestria, as crianças madeirenses apresentam percentagens de mestria mais baixas do que os seus pares norte-americanos(21) na corrida, salto a pé coxinho, salto e salto em comprimento sem corrida preparatória. Percentagens mais elevadas foram observadas no galope e no deslocamento lateral. Estes resultados são algo 'estranhos', porque o galope e o deslocamento lateral combinam vários movimentos básicos, nomeadamente, o andar e o salto ${ }^{6}$, 14). Ao nível das habilidades de manipulação, as crianças madeirenses apresentam valores percentuais mais baixos na quase totalidade das habilidades motoras. A única excepção foi observada no drible. Um cenário idêntico de resultados é encontrado na percentagem de mestria para cada componente/critério em cada habilidade de locomoção ou de manipulação. As crianças portuguesas apresentam percentagens de mestria inferiores aos seus pares norte-americanos(21) nas componentes/ critérios dois e três (corrida), um e quatro (galope), um, dois e três (pé coxinho), dois e três (salto), um, dois e quatro (salto em comprimento sem corrida preparatória). Valores percentuais similares entre as duas amostras são encontrados nas componentes/critérios um e quatro (corrida), três (galope), um (salto a pé coxinho) e dois, três e quatro (deslocamento lateral).

Resultados de mestria mais elevados nas crianças portuguesas são observados nas componentes/critérios dois (galope), cinco (salto a pé coxinho), três (salto em comprimento sem corrida preparatória) e um (deslocamento lateral).

Para as habilidades de manipulação, a análise comparativa é a seguinte: valores percentuais mais baixos dos portugueses nas componentes/critérios três, quatro e cinco (batimento de uma bola estática), um (drible), um e três (agarrar), um, dois e três (pontapear), um (lançamento por cima do ombro) e dois e quatro (lançamento por baixo). Valores de mestria similares são observados nas componentes/critérios um (batimento de uma bola estática), dois e quatro (drible), dois (agarrar), quatro (pontapear), dois, três e quatro (lançamento por cima do ombro) e um (lançamento por baixo). Percentagens mais elevadas dos portugueses comparativamente aos norte-ameri- canos são encontradas nas componentes/critérios dois (batimento de uma bola estática), três (drible) e três (lançamento por baixo).

As conclusões da presente pesquisa são várias: 1) as crianças madeirenses apresentam uma melhoria de resultados com a idade, na quase totalidade das habilidades motoras; 2 ) os rapazes são mais proficientes do que as raparigas nas habilidades de manipulação; 3) o maior número de crianças madeirenses foi classificado na categoria 'médio', nas habilidades de locomoção e nas habilidades de manipulação; 4) as crianças madeirenses apresentam equivalentes etários abaixo da média nas habilidades de locomoção e de manipulação; 5) um aumento de mestria com a idade é observado na corrida, galope, deslocamento lateral, drible, agarrar e lançamento por cima; 6) o dimorfismo sexual nas habilidades de locomoção é paralelo a outras pesquisas; 7) as crianças madeirenses são, na sua maioria, classificadas nas categorias 'média', 'abaixo da média', 'fraco' e 'muito fraco', colocando-as a um nível inferior às norteamericanas; 8) as crianças madeirenses apresentam percentagens de mestria mais baixas dos que os seus pares norte-americanos na quase totalidade das habilidades de locomoção e de manipulação; 9) valores percentuais de mestria mais elevados das crianças madeirenses comparativamente às norte-americanas são observados no galope e no deslocamento lateral; 10) as percentagens de mestria por componente/critério da amostra madeirense são, na generalidade, mais baixas nas crianças madeirenses comparativamente às norte-americanas.

A presente pesquisa fornece dados cruciais aos pais, professores, agentes desportivos e políticos em geral, no sentido de melhor interpretarem o significado associado à variação que ocorre no crescimento físico humano e no desenvolvimento motor da criança madeirense. O "espaço familiar", a escola e outras instituições de utilidade pública são o palco privilegiado para possíveis intervenções a este nível. Estratégicas sinérgicas para elevar o nível das habilidades de locomoção e de manipulação devem estimular a prática regular e sistemática de actividades de recreação, lazer e desportivas. 


\section{AGRADECIMENTOS}

O projecto de investigação 'Crescer com Saúde na Região Autónoma da Madeira' foi co-financiado pelo Governo da Região Autónoma da Madeira, através da Secretaria Regional de Educação e Cultura e da Secretaria Regional dos Assuntos Sociais, bem como pelo Programa Operacional Pluri-Fundos da RAM III, vertente Fundo Social Europeu e gerido pelo Centro de Ciência e Tecnologia da Madeira.

\section{CORRESPONDÊNCIA}

Duarte Luís de Freitas

Universidade da Madeira, Departamento de

Educação Física e Desporto

Campus Universitário da Penteada

9000-390 Funchal

Portugal-Madeira

Tel. 291705332

Fax. 291705339

E-mail:dfreitas@uma.pt 


\section{REFERÊNCIAS}

1. Andrade VM, Lima DA, Marques I (2006b). Comparação do desempenho de habilidades locomotoras e manipulativas em crianças de 7 e 8 anos de idade, de acordo com o género. Anais do III Congresso Brasileiro de Comportamento Motor. Rio Claro.

2. Andrade VM, Lima DA, Bastos CB, Marques I (2006a). Análise de habilidades locomotoras e manipulativas em crianças de 4 a 6 anos de idade. Anais do XV Encontro Anual de Iniciação Científica e Encontro de Pesquisas da UEPG. Ponta Grossa.

3. Branta C, Haubenstricker J, Seefeldt V (1984). Age changes in motor skills during childhood and adolescence. In: Terjung RL (ed.). Exercise and Sport Science Reviews, 12: 467520, Toronto: The Collamore Press

4. Centers for Disease Control and Prevention (2000). Physical activity and good nutrition. U.S. Department of Health and Human Services. National Center for Chronic Disease Prevention and Health Promotion. Atlanta.

5. Freitas DL, Maia JA, Rodrigues AL, Silva CA, Faria CA, Almeida MJ, Lopes VP, Rodrigues AJ, Neves AT, Antunes AM, Esteves CM, Afonso GA, Conceição LP (2006). Crescer com saúde na RAM - Um estudo no ensino préescolar e ensino básico. Painel Desportivo, 20: 9-12.

6. Gabbard CP (2004). Lifelong motor development. San Francisco: Pearson, Benjamin Cummings.

7. Lopes L (2006). Actividade física, recreio escolar e desenvolvimento motor. Estudo exploratório em crianças do $1^{\circ}$ ciclo do ensino básico. Dissertação apresentada a provas de mestrado. Braga: Universidade do Minho.

8. Malina R (1975). Anthropometric correlates of strength and motor performance. In: Wilmore H, Keogh F (ed.), Exercise and Sport Science Reviews, 3: 249-274, New York: Academic Press.

9. Malina RM (1984). Physical activity and motor development. performance in populations nutritionally at risk. In Pollitt E, Amante P (ed.), Energy and Intake and Activity. New York: Alan RL, 285-302.

10. Malina RM, Bouchard C, Bar-Or O (2004). Growth, maturation and physical activity. Champaign, IL: Human Kinetics.
11. Morris AM, Williams JM, Atwater AE, Wilmore JH (1982). Age and sex differences in motor performance of 3 trough 6 year old children. Res Q Exerc Sport, 53(3): 214-221.

12. Nelson JK, Thomas JR, Nelson KR (1986). Gender differences in children's throwing performance: biology and environment. Res Q Exerc Sport, 57(4): 280-287.

13. Nelson KR, Thomas JR, Nelson JK (1991). Longitudinal change in throwing performance: gender differences. Res $Q$ Exerc Sport, 62(1): 105-108.

14. Payne GV, Isaacs LD (2005). Human motor development: $A$ lifespan approach. New York: McGraw-Hill.

15. Safrit M (1990). Introduction to measurement in physical education and exercise science. Second edition. St. Louis: TimesMirror.

16. Saraiva L, Santos S, Mendes, Rodrigues L (2007). Validação do 'Test of Gross Motor Development - 2' (TGMD2): um estudo preliminar de validação com crianças portuguesas do $1^{\circ}$ ciclo de ensino básico. Comunicação apresentada no $3^{\circ}$ Seminário Internacional 'Educação Física, Lazer e Saúde Novas Realidades, Novas Práticas. Instituto de Estudos da Criança. Braga: Universidade do Minho.

17. SAS Institute (1990). SAS/STAT User's Guide. Cary, NC: SAS Institute.

18. Sinclair CB (1971). Movement and movement patterns of early childhood. Richmond VA: State Department of Education.

19. Thomas JR, French KE (1985). Gender differences across age in motor performance: a meta-analysis. Psychol Bull, 98(2): 260-282.

20. Thomas RJ, Nelson JK, Church G (1991). A developmental analysis of gender differences in health-related physical fitness. Pediatric Exercise Science, 3: 28-42.

21. Ulrich AD (2000). Test of gross motor development. Examiner's manual. Second edition. Austin: Pro-ed.

22. Wolfram Research (2003). Mathematica. version 5.0. Champaign, IL: Wolfram Research, Inc.

23. Zahner L, Dossegger A (2004). Motor activity-the key to development in childhood. In: Dossegger (ed.). L.Z.U.P.C.S.J.S.A. Active childhood-healthy life. Basle: Institute for Exercise and Health Sciences. 